\title{
Waste disposal facilities monitoring based on high-resolution information features of space im- ages
}

\author{
Maretta Kazaryan ${ }^{l}$, Arsen Simonyan ${ }^{2 *}$, Simon Simavoryan ${ }^{2}$, Elena Ulitina ${ }^{2}$, \\ and Rafik Aramyan ${ }^{3}$
}

${ }^{1}$ North Ossetia State Medical Academy, 40 Pushkinskaya., Vladikavkaz, 362019, Russia

${ }^{2}$ Sochi State University, 94, Plastunskaya, Sochi, 354003, Russia

${ }^{3}$ Russian-Armenian University, 123, Ovsepa Emina, Yerevan, 0051, Armenia

\begin{abstract}
In the article there is represented and solved the problem of space images recognition for the presence of solid household and industrial waste without binarization. The methods of stochastic geometry and mathematical analysis are used. In the work there is proposed an algorithm based on a trace transformation using discrete orthogonal transformations (DOT) to minimize the attribute space and carry out studies on correctness by Tikhonov. For the implementation of the algorithm there are used elements of mathematical analysis, wavelet analysis, functional analysis, theory of discrete orthogonal transformations, methods for deciphering space images in the problem of stochastic scanning of space images based on the formation of a triplet attribute with minimization of attribute space using DOT. The development a trace matrices and the selection of informative features by stochastic geometry to find WDF from high-resolution space images are investigated from the point of view of DOT apparatus application. A study of the sustainability task was also performed. The proposed technique was tested using the example of space photographs with a WDF image. Conclusions are drawn on the use of the method proposed in this article for the task of automatic computer generation and selection of informative features for determining waste disposal facilities from high-resolution space images. It is proposed to use the Tikhonov regularization method to introduce stability in this task.
\end{abstract}

\section{Introduction}

Waste disposal facilities (WDF) and household waste in general attract close attention in all countries of the world, regardless of their economic development.

Studies carried out in Europe [1, 2, 4] have shown that household waste, as a result of high consumption, represents some dynamic process and, in fact, they "live their life", i.e. it is a rather complex object in its chemical, morphological and molecular composition [1].

The situation is not the best in Russia. In general, the situation with landfills can be analyzed by only those that are removed centrally from urban garbage cans. Many spontaneous

\footnotetext{
${ }^{*}$ Corresponding author: oppm@mail.ru
} 
landfills are formed from incorrect attitude to this problem both at the household and at the state level.

Our State has transferred the waste removal to the population. The emergence of unauthorized WDFs is a consequence of people's unorganized activities. These landfills are a source of the environment contamination, deterioration of sanitary and epidemiological condition. The formation of a landfill is defined by a location, namely by a reference object, a strategic location, a reference to a geographical location, etc.; and time - time of year, time of day, start and end of formation, etc. The spontaneous nature of a landfill is determined by the predictability of its formation "in the direction" to the past, present or future. Occasional landfills are the most "spontaneous", since the place and the time of their formation is difficult to foresee and predict, it is unlikely and most accidental, sometimes it is difficult to detect by one or another method of environmental monitoring. In particular, episodic landfills are formed near buildings, in suburban recreation areas, in forest parks and forest parks massifs, on forest basins, etc. WDF can have various components in its composition [1,2].

Consider as an example the landfill in the village of Kuchino (Moscow region) (Figure $1)$.

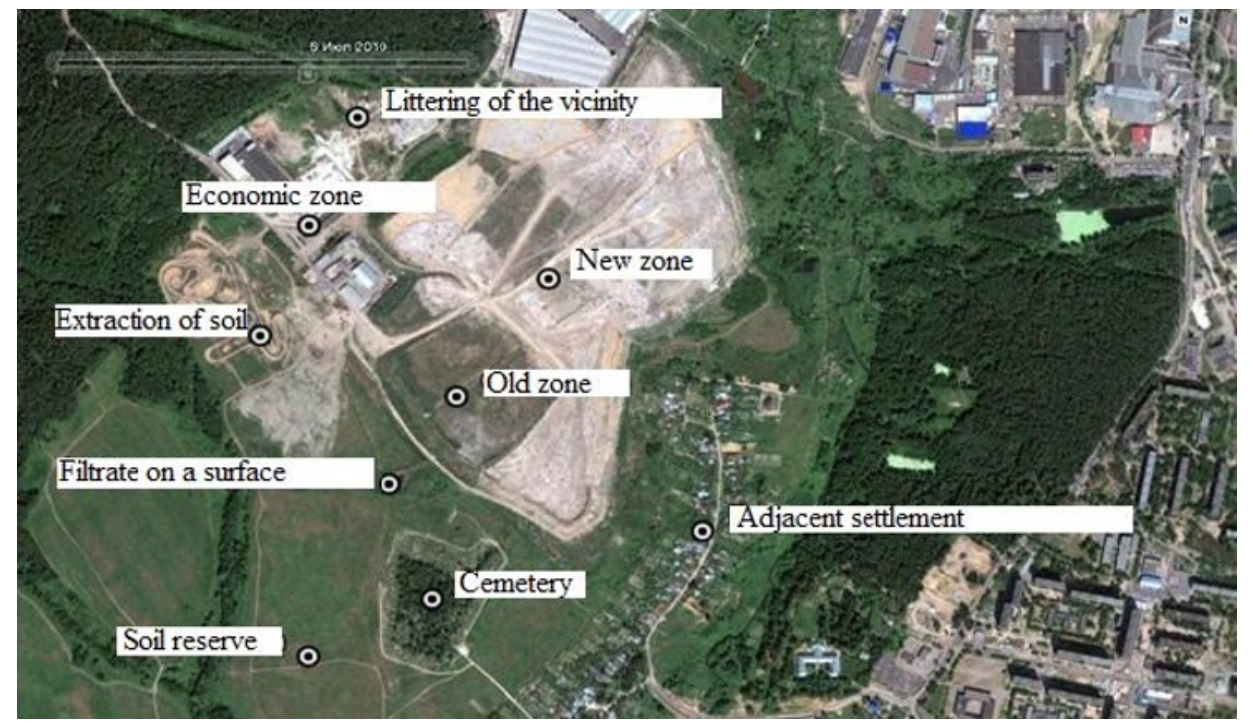

Fig. 1: Main components of WDF on the example of solid household waste landfill (SHW) "Kuchino"

This polygon includes: economic zone, storage area (old and new zones), expansion zone, structural elements (serpentine, slope, road path, etc.), relief elements (pit, half-pool, cover, etc.). Its surrounding natural environment includes: zones of soil extraction and soil reserve, adjacent settlement (village, city, etc.), forest and field massif, access road, cemetery, water source.

The inhabited locality is adjacent to WDF or is spaced some distance from it. The cemetery can be located near large WDF. In the old storage area there was no waste placement for a long time, in the new one it is carried out in the current period of time. The expansion zone is a part of the WDF territory, planned for waste disposal recently, and formerly referred to the surrounding natural environment.

Landfills monitoring by traditional methods is difficult due to their large number.

WDF monitoring based on remote sensing of the earth has advantages in this regard. It can be carried out in hard-to-reach places without direct contact with the territory under study. 
Certain techniques have been developed for space monitoring of solid household waste (SHW) and industrial waste (IW) [1, 2, 3, 5, 6, 7]. In particular:

- Method of automatic detection and estimation of parameters using discrete orthogonal transformations,

- A technique that checks the state of soil and vegetation,

- A procedure for checking the state of the underlying surface,

- Procedure for fractal and textural analysis of space images of landfills.

Developments carried out in the field of decryption of space images and their further use in monitoring implied preliminary simplification of the image, i.e. conversion to a binary view. This approach leads to the loss of information about the area under study.

\section{Materials and methods}

The main purpose of the study is to analyze and recognize space images during monitoring for WDF presence in another way. The mathematical basis of the work is the apparatus of stochastic geometry, functional analysis and integral geometry during considering the task of recognizing halftone and color images of WDF.

The subject of research is one of the technologies of operation of automatic space monitoring system for the presence of waste disposal facilities (WDF). This technology is based on the ideas of stochastic geometry, as well as geometric probability and covariogram.

The research tool is an algorithm based on trace transformation using discrete orthogonal transformations (DOP) to minimize the attribute space, as well as conducting studies on correctness by Tikhonov. The work uses elements of mathematical analysis, wavelet analysis, functional analysis, theory of discrete orthogonal transformations, methods of decryption of space images in the task of stochastic scanning of space images based on formation of a triplet feature with minimization of the feature space with the help of DOP.

At the same time, it is assumed that the original images have passed the proper stages of preliminary processing, there is no need to consider special procedures for validation of space images in the framework of the given studies.

\section{Analysis of Results}

Before we will go to the task of creating a feature space during decrypting space images, we turn to the technique of representing WDF as a certain geometric object.

There are many regularities of 3D-4D models of the Earth's surface and their spatialtemporal distribution of general and private nature in terms of space monitoring. Objects on the surface of the Earth are distributed according to certain laws and have a periodic structure at different levels of detailing. The periodicity of the surrounding world is observed not only on space, but also on other types of images. This makes it possible to apply algebra of geometric objects (multiplication of an object by a factor, grid multiplication of an object by an object, decomposition of the object into simple and compound multipliers, etc.) for their geometric calculus. The geometric programming approach based on algebra of geometric objects allows to solve many problems on automation of three-dimensional modeling, both in "statics" and in "dynamics". In particular, the approach allows to model scenes of the Earth 's surface according to the data of space and undersatellite monitoring in an automated and even automatic mode. This possibility is due to many prerequisites, for example, standardization of objects of the Earth 's surface of man-made and even natural origin (buildings, structures, roads, forest areas, etc.), periodization of the structure of natural and man-made objects at different levels of detailing (electric trains, railways and roads, their subsystems and different level of differentiation, etc.). 


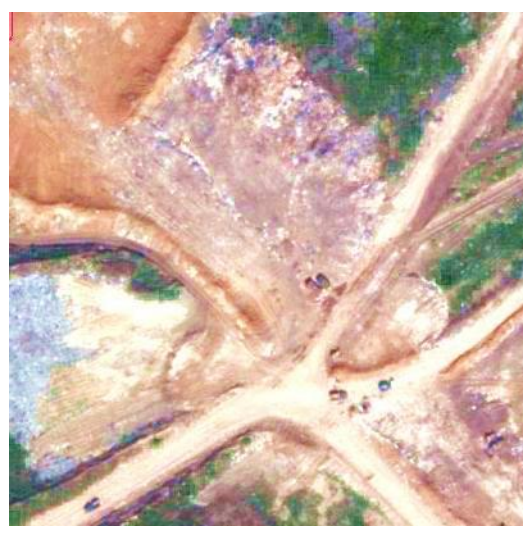

Fig. 2: Original WorldView2 image (the example SHW "Kuchino" polygon territory fragment)

Detailed studies in this direction are given in [14].

The decryption of space images by stochastic geometry has certain advantages and one of them is the ability to approach to the nature of human recognition, the essence of which is stochastic scanning. Stochastic scanning using integral geometry is based on a formula from integral geometry to determine the length of a planar curve using points of its intersection with straight lines $[9,10]$.

Thus, if we consider some finite field of random straight lines and then for each line we calculate the number of points of intersection the curve with each line, then we can approximately, as the arithmetic mean of the numbers, determine the length of the curve (in our case the size of the area filled with waste). To do this, we use the formula from integral geometry. Obviously, it is easier to calculate the number of points of intersection than the length of the curve. Indeed, this is important, as we can then determine the length of the perimeter of the area, which is filled with waste and garbage, that is, we get the exact size of the area we want to estimate. Recognition by this method has the property of independence from orientation and parallel displacement of the object under study. This is a valuable property because the objects themselves can be turned and moved in the image field.

Let $\mathrm{D}$ be WDF. In general, we will assume that $\mathrm{D}$ is a convex body within a sliding window having an arbitrary shape. For definiteness, we will consider a round window, respectively, with the attributes of this geometric figure. With regard to this assumption, we will consider formulas from [9] and related concepts, in particular the covariogram [10-12, 15$18]$.

Let $R^{n}(n \geq 2)$ is n-dimensional Euclidean space, $D \subset R^{n}$ is bounded convex area with internal points, and $V_{n}(\cdot)$ is $n$ - Lebesgue measure in $R^{n}$.

Definition 1 [26]. Function $C(D, h)=V_{n}(D \cap(D+h)), \quad h \in R^{n} \quad$ is called a body covariogram D. Here $D+h=\{x+h, x \in D\}$.

Matteron [10] has formulated the hypothesis that the covariogram of the convex body $\mathrm{D}$ defines it in the class of all convex bodies with accuracy to parallel transfers and reflections.

G. Bianchi and G. Averkov [11] have proved that each flat area is defined in the class of all flat convex areas by covariogram accurate to parallel transfers and reflections.

However, for $n>3$, the body is not defined by the covariogram, and in the case of $n=3$, the problem is still not solved (open problem). However, we believe that in the case of $n=3$ it is possible to use convex covariograms as its characteristic. 
The technique of decoding pictures by the methods of stochastic geometry is based on formation of triplet feature space $[8,15]$ with the help of trace transformations and minimization of feature space by means of discrete orthogonal transformations (DOP). Tikhonov [13] also has investigated this task of correctness.

We will follow the approach of automatically generating characteristics during generating a triplet characteristic. This makes it possible to form a characteristic space without expert involvement, i.e. the process takes place in automatic mode. Optimal elements are selected from the whole set of generated characteristic space, i.e. preliminary processing of the characteristic space is performed. Then the classic recognition algorithms with applying a learning set can be used.

Definition 2 [15]: let $l$ is a segment of a straight line; $\rho(\rho \geq 0)$ is a perpendicular drawn through the origin of coordinates up to intersecting with this line; $x, y$-are the coordinates in Cartesian system, $0 \leq \theta \leq 2 \pi$; then the representation of the line in the polar coordinate system has the form:

$$
l(\theta, \rho)=\{(x, y) \mid \rho=x \cos \theta+y \sin \theta\} .
$$

The method is generated by two stages.

The first step is the formation of a trace transform or a trace matrix. This conversion is obtained from scanning the image along this path. Discrete scanning array is generated, on the basis of which discrete trace transformation is built.

Consider the first step of a trace transformation generation. Let on the plane $(x, y)$ the image function $F(x, y)$ is given. Let $t$ be a point on a scanning line with a given covariogram, $\theta$ and $\rho$ be the normal coordinates of a straight line $l(\theta, \rho, t)$, that is defined by the equation: $x \cdot \cos \theta+y \cdot \sin \theta=\rho$. The analyzed image intersects with the scanning line. It is the function of two arguments $\mathrm{g}(\theta, \rho)$ considered as action of functional $\mathrm{T}$ on result of crossing: $g(\theta, \rho)=T(F \cap l(\theta, \rho, t))$ (the number of the scanning grating straight line intersections with the image).

Considering the discrete case, we have two discrete sets as the parameters of the scanning straight line

$$
\Omega=\left\{\theta_{1}, \theta_{2}, \ldots, \theta_{n}\right\} \text { and } \Gamma=\left\{\rho_{1}, \rho_{2}, \ldots, \rho_{n}\right\}
$$

Under the influence of functional $T$ we get a trace matrix:

$$
t_{i j}=T\left(F \cap l\left(\theta_{j}, \rho_{j}, t\right)\right)
$$

For definiteness of our research, we will consider halftone images, i.e. black and white images. The trace matrix constructed according to this method is determined primarily by the numerical characteristics of the section of the image with scanning straight line (number of intersections, sum of lengths of intersection areas, etc.), as well as information on the vicinity of the section of the scanning straight line.

Conversion of columns of the obtained matrix is carried out by means of diametrical functional $P$.

Let

$$
P=\sqrt{\sum_{i=1}^{m} g^{2}\left(\theta_{j}, \rho_{i}\right)} \quad i=1, \ldots, m ; j=1, \ldots n \text {. }
$$

Let

$$
h(\theta)=P(g(\theta, \rho))
$$

As a result, we have $2 \pi$ - periodic curve or a vector for considering discrete case. 
Performing the vector compression by means of the functional $\Theta$, we get a number as the feature for a given image.

Let

$$
\Theta=\frac{\max _{j} h\left(\theta_{j}\right)}{\min _{j} h\left(\theta_{j}\right)}
$$

So, a triplet feature is obtained as a sequential application of three functionals

$$
\Pi(F)=\Theta \circ P \circ T(F \cap l(\theta, \rho, t)) .
$$

In case of triplet features generating, geometric and other a priori characteristics of the obtained features are not taken into account.

After that, informative characteristics are minimized. The article proposes to apply the apparatus of orthogonal transformations and to research this task on sustainability.

The algorithm is rather universal. Its idea is that: in order to recognize space images, it is necessary to generate many features and then to choose from them optimal ones by the appropriate criterion. Therefore, when an automatic system is built, it is necessary to perform recognition system learning with allowance for the outgoing from this costs. However, at the same time, when territories are investigated and monitoring for solid household waste is carried out, in rare cases it is possible to know the geometric characteristics of WDF in advance. This is important for classifying the object under investigation.

The second step is to minimize feature space or compression.

In general, the Karhunen-Loeve transformation is used in theoretical studies to determine the minimum set of informative search features. But in practice this orthogonal transform is practically not used because it not has a fast algorithm. Therefore, it is advisable to take advantage of a series of DOPs that have fast algorithms and have been tested for several decades. The application of orthogonal transformation brings some noise when one is working with space images and conducting environmental analysis of the investigated territories for the presence of WDF. Therefore, to eliminate incorrectness of the task of compressing the feature space, we will use the Tikhonov method [13]. After preprocessing the source information, one can go to traditional image recognition using the standard algorithm.

\section{Discussion}

Certain requirements must be met for the experiment with space images showing landfills. These are geographical, geometric and technological requirements [1,2]. We will use the following software tools to conduct the experiment:

- GIS GoogleEarth. Using it, the Earth 's surface is placed on the Internet and it is possible to pick out satellite images with sufficiently good characteristics, i.e. suitable images will have a high resolution;

- Matlab and ObjectPascal for calculations and implementation of the proposed algorithm in the program form.

Landfill images have a number of decryption features. They make it possible to discriminate and monitor WDF on a space image. Let list these features that have the random character: the contour of the object, the distribution of parts of the surface object, the spatial distribution of the normalized WDF texture signal; spatial linking to natural and man-made objects (roads, shops, garages, etc.); multi-component structure, etc.

Main decryption features of landfill are shape and texture $[1,2,8]$. Let us describe the subject area, namely landfills $[1,2]$. We are interested in the description of landfills in geometric and textural terms. The landfill has a rough surface, which can be accompanied by 
shielding elements. Debris can be mixed with sand, vegetation, condensed earth, etc. A garbage dump has a random spatial distribution of brightness on different ranges of the spectrum. Further detailed description of landfill characteristics is described in monographs $[1,2]$. Let us take the halftone fragment of the garbage dump as the original image.

We shall build features that characterize the brightness and geometric features of the image.

In this connection consider two groups of triplet features: geometric features and luminance features. They are triplet features of type (1). Let the length of the line be used to draw geometric features. Let the average brightness of the segment be used to form a plurality of features by which the brightness of the image is characterized.

Algorithm for determining the boundaries of the luminance-uniform segments obtained as a result of the line $l(\rho, \theta)$ scanning is the following:

Step 1. Let scanning be along the straight line $l(\rho, \theta)$, then determine the brightness at each of its points;

Step 2. Generation of brightness function $I(x)$ for scanning line $l(\rho, \theta)$;

Step 3. Calculation the derivative of brightness function $d I(x) / d x$.

We define brightness differences as points of extremes, which are the boundary points of segments of the scanning straight line $l(\rho, \theta)$.

Figure 3 shows the algorithm for the attribute vector obtaining.

Let us select the most informative elements of the obtained attribute vector using a DOP apparatus. For definiteness we examine the wavelet transformation and the possibility of selecting the most informative elements of the attribute vector by means of this transformation. The task of selecting informative characteristics with the application of the Wavelet-Haar transformation was analyzed in sufficient details in the work [6].

Let

$$
F=\left\|\varphi_{j}(i)\right\|_{j, i=0, N-1}
$$

be the Wavelet-Haar matrix and let $\bar{x} \in X_{\Delta}$,

where $\mathrm{X}_{\Delta}=\left\{\bar{x}=\left(x_{0}, \ldots, x_{N-1}\right): \max _{\mathrm{k}}\left|x_{k-1}-x_{k}\right| \leq \Delta\right\}$.

Consider the following optimization task to determine the most informative elements. Required to find $y_{j} \rightarrow$ extr under condition $\bar{x} \in X_{\Delta}$, where

$$
y_{j}=x_{0} \varphi_{j}(0)+x_{1} \varphi_{j}(1)+\ldots+x_{N-1} \varphi_{j}(N-1)
$$

Using the principle of optimality in dynamic programming, we obtain the following estimate:

$$
\max _{x \in X_{\Delta}}\left|y_{j}\right|=\Delta \sum_{m=1}^{N-1}\left|\sum_{i=0}^{m-1} \varphi_{j}(i)\right| \quad, \quad j=\overline{1, N-1}
$$




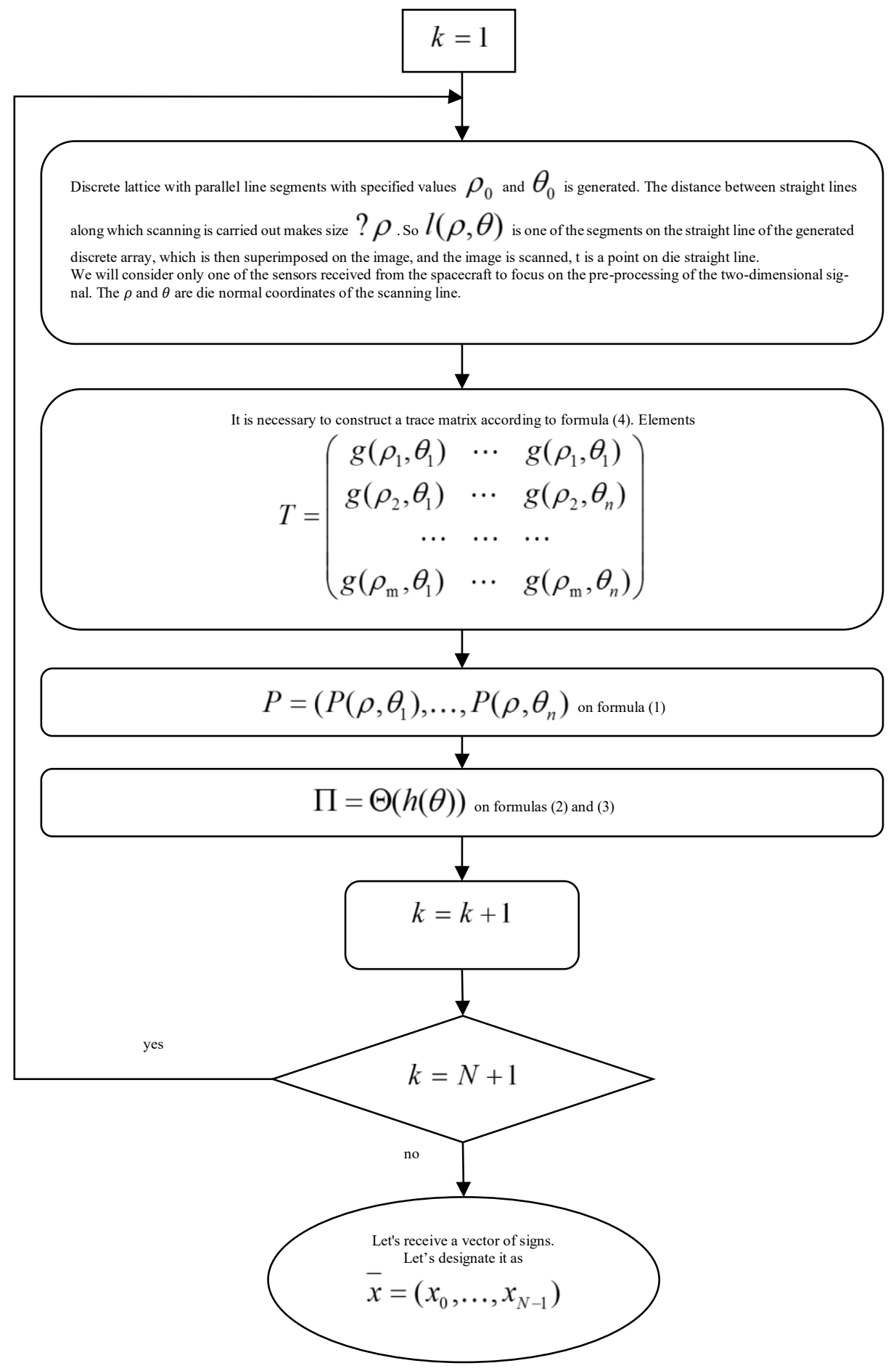

Fig. 3: Algorithm for the attribute vector obtaining 


\section{Statement}

The least informative elements that can be zeroed during further work are the elements of the last pack of the vector $\bar{y}$.

\section{Proof.}

Let $F=F_{H}(N)$

be a matrix of order $N=2^{n+1}(n \geq 0), \bar{x} \in X_{\Delta}, \bar{y}=F_{H}(N) \bar{x}=\left(y_{0}, \ldots, y_{N-1}\right)^{T}$.

Based on (2) and from the form of the wavelet-Haar matrix, we have for $j=2^{k-1}, \ldots, 2^{k}-1$ :

$$
\begin{aligned}
& \frac{1}{\Delta} \max _{x \in X_{\Lambda}}\left|y_{j}\right|=\frac{1}{\sqrt{N}}\left[\sqrt{2^{k-1}}+2 \sqrt{2^{k-1}}+\ldots+2^{n+1-k} \sqrt{2^{k-1}}+\left(2^{n+1-k}-1\right) \sqrt{2^{k-1}}+\left(2^{n+1-k}-2\right) \sqrt{2^{k-1}}+\ldots+\sqrt{2^{k-1}}\right]= \\
& =\frac{\sqrt{2^{k-1}}}{\sqrt{N}}\left[1+2+\ldots+2^{n+1-k}+\left(2^{n+1-k}-1\right)+\ldots+1\right]=\frac{\sqrt{2^{k-1}}}{\sqrt{N}} 2^{2(n+1-k)}=\frac{N^{3 / 2}}{\sqrt{2^{3 k+1}}}
\end{aligned}
$$

From here follows that the least informative elements are the elements of the last packs of the vector $\bar{y}$, therefore, they can be set equal to zero during further processing of the image including space image (SI).

Using this statement, let us conduct an experiment. So, for experimental confirmation of the above theoretical calculations, consider the task of compression or zone coding of a signal (image) as a stage of image preprocessing.

The mathematical formulation of the compression problem with regularization [13] and without regularization is given below.

Let $\bar{x}=\left(x_{0}, \ldots, x_{N-1}\right)$ be an initial feature vector $N ; F$ be a discrete orthogonal transformation (Walsh, Fourier, Haar transformation, and so on), $F^{-1}$ be inverse transformation, $S$ be a matrix for selection of dimension $m \times N$ with rank $m, 1 \leq m \leq N, R_{\alpha}$ be a regularizing matrix in the problem of compression of the initial vector $\bar{x}$ defined as follows:

$$
\boldsymbol{R}_{\propto}=\left[\begin{array}{ccc}
\varphi(1, \alpha) & \cdots & \mathbf{0} \\
\vdots & \ddots & \vdots \\
0 & \cdots & \varphi(N, \alpha)
\end{array}\right]
$$

where $\varphi(n, \alpha)$ are regularizing factors; $n=\overline{1, N} ; \alpha$ is a regularizing parameter.

The problem is in choosing, for given $F_{0}, S_{0}$, such regularizing matrix $R_{\alpha}$, that the following condition will be satisfied: $\rho\left(\bar{x}, R_{\alpha} \widehat{x}\right) \rightarrow \min$, where $\rho$ is the given metric;

$$
\widehat{x}=F_{0}^{-1} S_{0}^{T} S_{0} F_{0} \bar{x}
$$

Note: for a fixed transformation $F_{0}$, an arbitrary matrix $S, R_{\alpha}=I$ this problem, known as the problem of zone coding by means of the transformation $F$, was studied in [6].

The form of the matrix $S$ determines which elements of the spectral space of the initial vector are redundant. So, if this is a wavelet-Haar transformation, it is necessary to discard 
the last elements of the transformation matrix, for the Fourier transform the central components of the spectrum of the initial signal are redundant, for discrete Walsh, Walsh-Paley transforms, the last elements of the spectral vector are uninformative (Fig. 4).

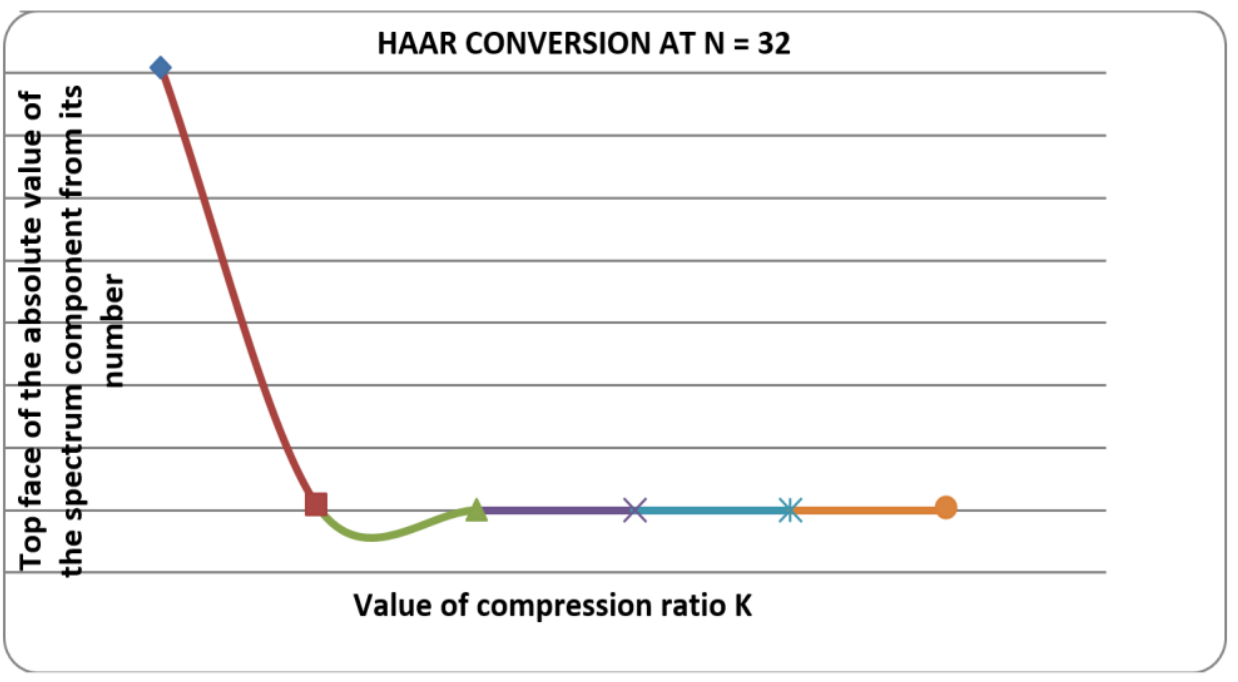

Fig.4. Dependence of the upper face of the absolute value of the spectral component on its number when applying the Wavelet-Haar transformatin

The values $\alpha$ и $\varphi(n, \alpha)$ are selected depending on the corresponding orthogonal transformation, on the input data, and on the structure of the transformations.

The form of the matrix $R_{\alpha}$ is used in the discrete interpretation of the problem on image compression with regularization, although it was defined for the continuous case $[5,13]$.

Consider the signal coding general algorithm with regularization, presented below.

\subsection{General algorithm for compressing one-dimensional signals with a regu- larizing matrix $r_{\alpha}$.}

Step 1. The vector signal $\bar{x}$ is transformed by means of $F: \bar{y}=F \bar{x}=\left(y_{0}, \ldots, y_{N-1}\right)^{T}$,

Step 2. By means of the operator $S$ the vector of components rapprochement $\delta y_{\delta}$ is transformed into a vector whose dimension is less than the dimension of the vector $\tilde{y}_{\delta}=\left(\tilde{y}_{0}, \ldots, \tilde{y}_{N-1}\right)$, whose dimension is less than the dimension of the vector that is to be transmitted over the communication channel, stored, etc. (the value of corresponding relation is called as compression coefficient).

Step 3. "Extrapolation" is performed by means of the $S T$, matrix, i.e. on the receiving side, the received vector is supplemented to dimension $N$ (so, for example, all components except the selected ones that are assumed to be zero).

Step 4. The received vector is subjected to inverse transformation $F^{-1}$.

Step 5. Multiplication by a matrix of regularizing factors is carried out $R_{\alpha}$.

As a result of the implementation of this algorithm, the restoration of the initial vector is carried out with errors: $\varepsilon_{1}=\rho_{C}\left(\bar{x}, F^{-1} S^{T} S F \overline{x_{\delta}}\right), \quad \varepsilon_{2}=\rho_{C}\left(\bar{x}, R_{\alpha} F^{-1} S^{T} S F \overline{x_{\delta}}\right)$, where $C$ is the uniform metric. 
The problem is in choosing $\varphi(n, \alpha)$ so that for a given $k$ the following condition is satisfied: $\varepsilon 2<<\varepsilon$.

In Fig. 5 are shown the compression errors with regularization and without regularization along the ordinate axis, for certain values of the compression coefficient $k$ along the abscissa axis for a given value of $\alpha=0,5$. It can be seen that $\varepsilon 2<<\varepsilon 1$, where $\varepsilon 2$ is the compression error with regularization and $\varepsilon l$ is the compression error without regularization.

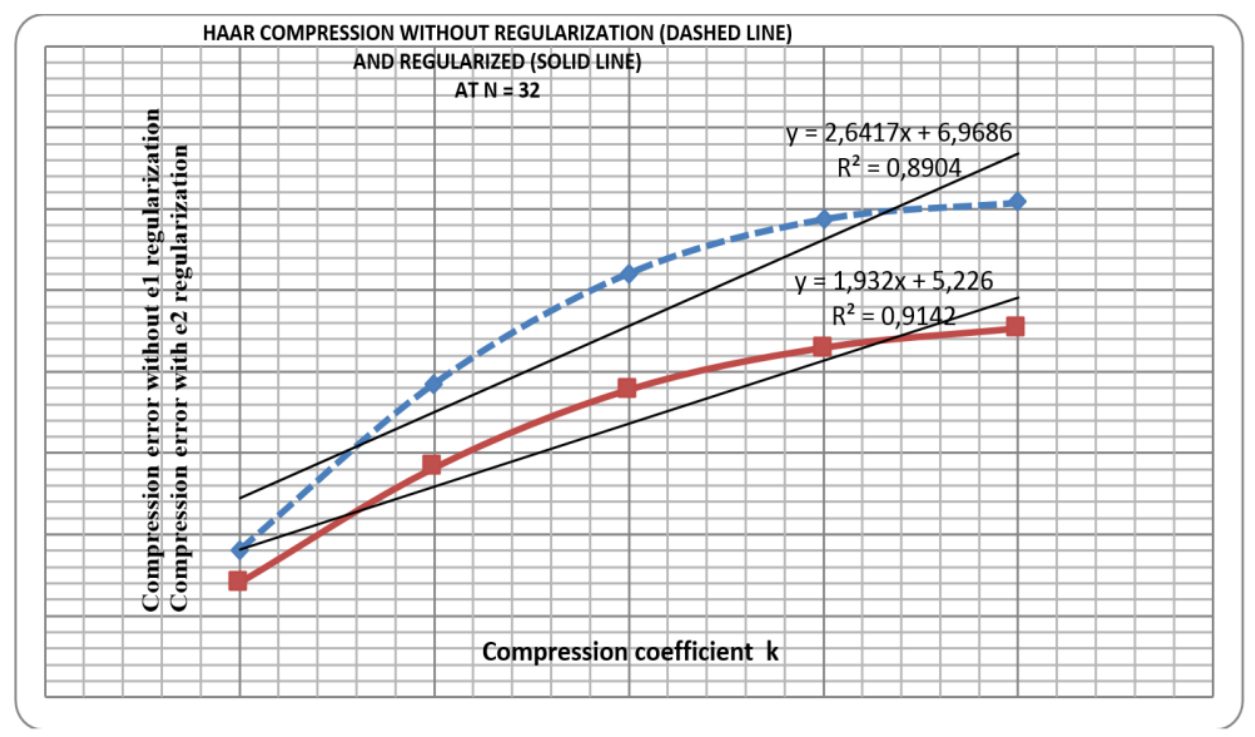

Fig. 5: Location of compression errors with and without regularization

Further, with reduced feature space, one can use ordinary algorithms for the recognition and decryption of space images $[1,2]$.

\section{Conclusion}

In the article it is proposed a method of expansion, and a significant feature space, during decrypting space images, allowing to carry out environmental monitoring for the presence of WDF objects. The features are generated, and this makes it possible to form them automatically without the participation of an expert.

Based on the results of the experiment, it can be concluded that the proposed method can be used for the task of automatic computer generation and selection of informative features for determination of waste disposal objects from high-resolution space images.

To optimize the characteristic space, one can use the DOP apparatus, and then check the stability of the algorithm. It is proposed to use the method of regularization of Tikhonov to introduce correctness in the task under investigation.

The reported study was funded by RFBR according to the research project №18-51-05010.

\section{References}

1. M.L. Kazaryan et al., Monografiya (Moscow, INFRA-M, 2019)www.dx.doi.org/10.12737/monografhy_5c4efa771779a4.89852001. 
2. M.L. Kazaryan et al., Monografiya (Moscow, INFRA-M, 2019). www.dx.doi.org/10.12737/monografhy_5cc2bd80eefd51.15862680.

3. M. L. Kazaryan, M. A. Schahramanian, A.A. Richter, Serbian journal of electrical engineering, 14(3), 343-364 (2017). DOI: https://doi.org/10.2298/SJEE1703343K

4. Improving Recycling Markets. (Paris: OECD, 2006)

5. M.L. Kazaryan, M.A. Shahramanian, S. Zabunov, Aerospace Research in Bulgaria, (Bulgarian Academy of Sciences. Space Research and Technology Institute), 31, (2019). DOI: https://doi.org/10.3897/arb.v31.e06

6. M.L. Kazaryan, M.A. Shahramanian, S. Zabunov, Aerospace Research in Bulgaria, (Bulgarian Academy of Sciences. Space Research and Technology Institute), 30, 96103 (2018) DOI: https://doi.org/10.3897/arb.v30.e09

7. M. Kazaryan, M. Shakhramanyan, A. Richter, R. Nedkov, D. Borisova, N. Stankova, I. Ivanova, M. Zaharinova, Proceedings of SPIE - The International Society for Optical Engineering. "Image and Signal Processing for Remote Sensing XXIII", 23, 2278572 (2017). DOI: $10.1117 / 12.2278572$

8. V.P. Fralenko Programmnye sistemy: teoriya i prilozheniya, 4(22), 19-39 (2014).

9. L.A. Santalo, Integral Geometry and Geometric Probability. (Addision-Wesley, MA, 2004).

10. ZH. Materon, Sluchajnye mnozhestva i integral'naya geometriya. (Mir, Moskva, 1978).

11. G. Bianchi and G. Averkov, Journal of the European Mathematical Society, 11, 11871202, (2009).

12. N.G. Agaronyan, V.K. Oganyan, Izvestiya NAN Armenii, 4(40), 43-56, (2005).

13. M.L. Kazaryan, Telecommunications and Radio Engineering, 70(18), 1631-1650 (2011) DOI: 10.1615/TelecomRadEng.v70.i18.30

14. A.A. Richter, M.L. Kazaryan, M.A. Shahramanian, V.V. Voronin, Proceedings of SPIE - The International Society for Optical Engineering, (France, 2019)doi: $10.1117 / 12.2534749$

15. W.S. Kendall, New perspectives in stochastic Geometry (Oxford, UK: Oxford University press, 2010).

16. A.G. Gasparyan, V.K. Ohanyan, Journal of Contemporary Mathematical Analysis, 48(3), 110-122 (2013).

17. A.G. Gasparyan, V.K. Ohanyan, Journal of Contemporary Mathematical Analysis, 49(4), 17-34 (2014).

18. V.K. Ohanyan, V.G. Bardakhchyan, A.R. Simonyan, E.I. Ulitina, Programmnye sistemy i vychislitel'nye metody, 2, 1-10 (2019). 